ISBN 978-93-84468-83-5

International Conference on Issues in Business, Economics, Marketing and Mathematics

(IBEMM-16)

Singapore Dec. 5-6, 2016

\title{
Business Cycles with Capital, Environment and Renewable Resources
}

\author{
Wei-Bin Zhang \\ Ritsumeikan Asia Pacific University, Beppu-shi, Japan
}

\begin{abstract}
This paper identifies business cycles in a dynamic economic model with endogenous physical capital, pollution, and renewable resources proposed by Zhang (2012). The model describes a dynamic interdependence among physical accumulation, environmental dynamics, resource change, and division of labor in competitive markets under government's intervention in environmental protection. The refined economic structure makes it possible to study the interactions among economic variables which are not found in the existing literature of economics in a single analytical framework with microeconomic foundation. This study generalizes Zhang's model by allowing all the time-dependent parameters to be time-dependent.
\end{abstract}

Keywords: business cycles, renewable resource, harvesting, environmental change, capital accumulation

\section{Introduction}

This paper identifies business cycles in a dynamic economic model with endogenous physical capital, pollution, and renewable resources proposed by Zhang [1]. This study attempts to provide another contribution to the literature by identifying economic fluctuations in an economic growth model by Zhang [1]. The paper is organized as follows. Section 2 generalizes Zhang's model. Section 3 shows how we solve the dynamics and simulates the model. Section 4 simulates the model to demonstrate business cycles due to some periodic shocks on the economic system. Section 5 concludes the study.

\section{The Basic Model}

This section is built on Zhang [1] by allowing all the time-dependent parameters to be time-dependent. The economy has three sectors and one government (which collects taxes for environmental protection). The three sectors are production, environmental and renewable resource sectors. Households own assets of the economy and distribute their incomes to consume and save. Production sectors or firms use labor and capital as inputs. Exchanges take place in perfectly competitive markets. We assume a homogenous and fixed population, $N$. The labor force is distributed among the three sectors. We select commodity to serve as numeraire. Let $N_{i}(t)$ and $K_{i}(t)$ stand for respectively labor and physical capital inputs. The production function $F_{i}(t)$ is specified as

$$
F_{i}(t)=A_{i}(t) \Gamma_{i}(E(t), t) K_{i}^{\alpha_{i}(t)}(t) N_{i}^{\beta_{i}(t)}(t), A_{i}(t), \alpha_{i}(t), \beta_{i}(t)>0, \alpha_{i}(t)+\beta_{i}(t)=1,
$$

where $A_{i}(t), \alpha_{i}(t)$, and $\beta_{i}(t)$ are positive parameters. Here, $\Gamma_{i}(E(t), t)$ is a function of the environmental quality measured by the level of pollution, $E(t)$. The marginal conditions are given by

$$
r(t)+\delta_{k}(t)=\alpha_{i}(t) \bar{\tau}_{i}(t) F_{i}(t) / K_{i}(t), w(t)=\beta_{i}(t) \bar{\tau}_{i}(t) F_{i}(t) / N_{i}(t),
$$

where $\delta_{k}(t)$ is the given depreciation rate of physical capital and $\tau_{i}(t)$ is the fixed tax rate, $\bar{\tau}_{i}(t) \equiv 1-\tau_{i}(t), 0<\tau_{i}(t)<1$. Let $X(t)$ stand for the stock of the resource. Let $\phi(t)$ stand for the maximum 
possible size for the resource stock $\phi_{0}(t)$ for "uncongested" or "intrinsic" growth rate of the renewable resource. The change rate in the stock is then equal to the natural growth rate minus the harvest rate

$$
\dot{X}(t)=\phi_{0}(t) X(t)(1-X(t) / \phi(t))-F_{x}(t) .
$$

Let $N_{x}(t)$ and $K_{x}(t)$ respectively stand for the labor force and capital stocks employed by the resource sector. We assume that harvesting of the resource is carried out according to the harvesting production function

$$
\begin{gathered}
F_{x}(t)=A_{x}(t) \Gamma_{x}(E(t), t) X^{b(t)}(t) K_{x}^{\alpha_{x}(t)}(t) N_{x}^{\beta_{x}(t)}(t), A_{x}(t), b \geq 0(t), \\
\alpha_{x}(t), \beta_{x}(t)>0, \alpha_{x}(t)+\beta_{x}(t)=1,
\end{gathered}
$$

where $A_{x}(t), b(t), \alpha_{x}(t)$ and $\beta_{x}(t)$ are parameters and $\Gamma_{x}(E(t), t)$ is a function of the environmental quality measured by the level of pollution. Let $p(t)$ and $\tau_{x}(t)$ respectively stand for the price of the resource and the fixed tax rate on the harvesting. We introduce $\bar{\tau}_{x}(t) \equiv 1-\tau_{x}(t), 0<\tau_{x}(t)<1$. The marginal conditions are given

$$
r(t)+\delta_{k}(t)=\alpha_{x}(t) \bar{\tau}_{x}(t) p(t) F_{x}(t) / K_{x}(t), w(t)=\beta_{x}(t) \bar{\tau}_{x}(t) p(t) F_{x}(t) / N_{x}(t)
$$

We specify the dynamics of the stock of pollutants as follows

$$
\dot{E}(t)=\theta_{i}(t) F_{i}(t)+\theta_{x}(t) F_{x}(t)+\bar{\theta}_{c}(t) C(t)+\bar{\theta}_{x}(t) C_{x}(t)-Q_{e}(t)-\theta_{0}(t) E(t),
$$

in which $\theta_{i}(t), \theta_{x}(t), \bar{\theta}_{c}(t), \bar{\theta}_{x}(t)$, and $\theta_{0}(t)$ are positive parameters and

$$
Q_{e}(t)=A_{e}(t) \Gamma_{e}(E(t), t) K_{e}^{\alpha_{e 0}(t)}(t) N_{e}^{\beta_{e 0}(t)}(t), A_{e}(t), \alpha_{e 0}(t), \beta_{e 0}(t)>0,
$$

where $N_{e}(t)$ and $K_{e}(t)$ are respectively the labor force and capital stocks employed by the environmental sector, $A_{e}(t), \alpha_{e}(t)$, and $\beta_{e}(t)$ are positive parameters, and $\Gamma_{e}(E(t), t)(\geq 0)$ is a function of $E(t)$.

We denote per capita wealth by $k(t)$, where $k(t) \equiv K(t) / N$. Let $\tau_{k}$ and $\tau_{w}$ respectively stand for the tax rates on the interest payment and wage income. Per capita current income is given by

$$
y(t)=\bar{\tau}_{k}(t) r(t) k(t)+\bar{\tau}_{w}(t) w(t) .
$$

where $\bar{\tau}_{k}(t) \equiv 1-\tau_{k}(t)$ and $\bar{\tau}_{w}(t) \equiv 1-\tau_{w}(t)$. The per capita disposable income is given by

$$
\hat{y}(t)=y(t)+k(t) \text {. }
$$

The budget constraint is

$$
\left(1+\tau_{c}(t)\right) c(t)+s(t)+\left(1+\tilde{\tau}_{x}(t)\right) p(t) c_{x}(t)=\hat{y}(t)
$$

where $\tau_{c}(t)$ and $\tilde{\tau}_{x}(t)$ are respectively the tax rates on the consumption of the goods and the resource. For simplicity of analysis, we specify the utility function as follows

$$
U(t)=\Gamma_{u}(E(t), t) c^{\xi_{0}(t)}(t) s^{\lambda_{0}(t)}(t) c_{x}^{\chi_{0}(t)}(t), \quad \xi_{0}(t), \lambda_{0}(t), \chi_{0}(t)>0,
$$

where $\xi_{0}(t)$ the propensity to consume, $\lambda_{0}(t)$ the propensity to own wealth, and $\chi_{0}(t)$ the propensity to consume the resource good. Maximizing $U(t)$ in (9) subject to the budget constraint (8) yields

$$
\begin{gathered}
c(t)=\xi(t) \hat{y}(t), s(t)=\lambda(t) \hat{y}(t), \quad p(t) c_{x}(t)=\chi(t) \hat{y}(t), \\
\xi(t) \equiv \rho(t) \xi_{0}(t) /\left(1+\tau_{c}(t)\right), \quad \lambda(t) \equiv \rho(t) \lambda_{0}(t), \quad \chi(t) \equiv \rho(t) \chi_{0}(t) /\left(1+\tilde{\tau}_{x}(t)\right), \quad \rho(t) \equiv 1 /\left(\xi_{0}(t)+\lambda_{0}(t)+\chi_{0}(t)\right) .
\end{gathered}
$$

According to the definition of $s(t)$, the change in the household's wealth is given by

$$
\dot{k}(t)=s(t)-k(t)
$$


The equation simply states that the change in wealth is equal to saving minus dissaving.

The government's tax income is

$$
Y_{e}(t)=\tau_{i}(t) F_{i}(t)+\tau_{x}(t) p(t) F_{x}(t)+\tau_{c}(t) C(t)+\tau_{x}(t) p(t) C_{x}(t)+\tau_{w}(t) N(t) w(t)+\tau_{k}(t) r(t) K(t)
$$

As there are only two input factors in the environmental sector, the government budget is given by

$$
\left(r(t)+\delta_{k}(t)\right) K_{e}(t)+w(t) N_{e}(t)=Y_{e}(t) .
$$

The government's optimal problem is given by

$$
\operatorname{Max} Q_{e}(t) \quad \text { s.t.: }\left(r(t)+\delta_{k}(t)\right) K_{e}(t)+w(t) N_{e}(t)=Y_{e}(t) .
$$

The optimal solution is given by

$$
\begin{gathered}
\left(r(t)+\delta_{k}(t)\right) K_{e}(t)=\alpha_{e}(t) Y_{e}(t), w(t) N_{e}(t)=\beta_{e}(t) Y_{e}(t), \\
\alpha_{e}(t) \equiv \alpha_{e 0}(t) /\left(\alpha_{e 0}(t)+\beta_{e 0}(t)\right), \quad \beta_{e}(t) \equiv \beta_{e 0}(t) /\left(\alpha_{e 0}(t)+\beta_{e 0}(t)\right) .
\end{gathered}
$$

The demand for and supply of the resource balance at any point of time

$$
c_{x}(t) N(t)=F_{x}(t)
$$

Let $N$ and $K(t)$ stand for respectively the labor supply and total capital stock. The labor force is allocated between the three sectors. As full employment of labor and capital is assumed, we have

$$
K_{i}(t)+K_{x}(t)+K_{e}(t)=K(t), \quad N_{i}(t)+N_{x}(t)+N_{e}(t)=N(t)
$$

We have thus built the dynamic model. We now examine dynamics of the model.

\section{The Dynamics and its Properties}

This section examines dynamics of the model. First, we introduce a new variable by $z(t) \equiv\left(r(t)+\delta_{k}(t)\right) / w(t)$. We now show that the dynamics can be expressed by 3 differential equations.

\section{Lemma}

The motion of the system is determined by the 3 differential equations

$$
\dot{z}(t)=\bar{\Lambda}_{z}(z(t), X(t), E(t), t), \quad \dot{X}(t)=\bar{\Lambda}_{X}(z(t), X(t), E(t), t), \quad \dot{E}(t)=\bar{\Lambda}_{E}(z(t), X(t), E(t), t),
$$

where the functions in (18) are functions of $z(t), X(t)$ and $E(t)$ given in the appendix. Moreover, all the other variables can be determined as functions of $z(t), X(t)$ and $E(t)$ at any point of time by the following procedure: $K(t)$ by $(\mathrm{A} 16) \rightarrow K_{i}(t)$ and $K_{x}(t)$ by (A13) $\rightarrow K_{e}(t)$ by (A11) $\rightarrow N_{i}(t), N_{x}(t)$ and $N_{e}(t)$ by (A1) $\rightarrow F_{x}(t)$ by (4) $\rightarrow F_{i}(t)$ by (1) $\rightarrow Q_{e}(t)$ by (7) $\rightarrow p(t), r(t)$ and $w(t)$ by (2) $\rightarrow k(t)=K(t) / N(t) \rightarrow \hat{y}(t)$ by $(8) \rightarrow$ $c(t), c_{x}(t)$ and $s(t)$ by (11).

We specify the parameter values as follows

$N_{0}=5, \alpha_{i}=0.32, A_{i}=1, \alpha_{x}=0.4, A_{x}=0.7, \alpha_{e 0}=0.6, A_{e}=0.3, \phi=3, \phi_{0}=5, \tau_{i}=0.02$,

$\varepsilon_{i}=0.6, \tau_{x}=0.04, \tau_{k}=0.01, \tau_{w}=0.01, \tau_{c}=0.04, \lambda_{0}=0.6, \xi_{0}=0.09, \chi_{0}=0.02, b=0.7$

$b_{i}=0.3, b_{e}=0.2, b_{x}=0.2, \theta_{i}=0.03, \theta_{x}=0.03, \bar{\theta}_{c}=0.03, \bar{\theta}_{x}=0.02, \theta_{0}=0.04, \delta_{k}=0.05$. (19)

Under (19), we identify the equilibrium values as follows

$$
\begin{gathered}
K=32.45, \quad E=1.69, \quad X=2.61, \quad F_{i}=6.31, \quad F_{x}=1.71, \quad Q_{e}=0.35, \quad N_{i}=4.22, \\
N_{x}=0.60, \quad N_{e}=0.18, \quad K_{i}=24.22, \quad K_{x}=4.89, \quad K_{e}=3.34, \quad p=0.61, \quad r=0.032,
\end{gathered}
$$




$$
w=1.00, c_{X}=0.34, c=0.94, s=6.49 .
$$

The three eigenvalues are calculated as: $\{-4.146,-0.188,-0.092\}$.

With $z(0)=0.06, Z(0)=1.5$, and $X(0)=2.4$, we plot the motion of the economic system in Figure 1 .
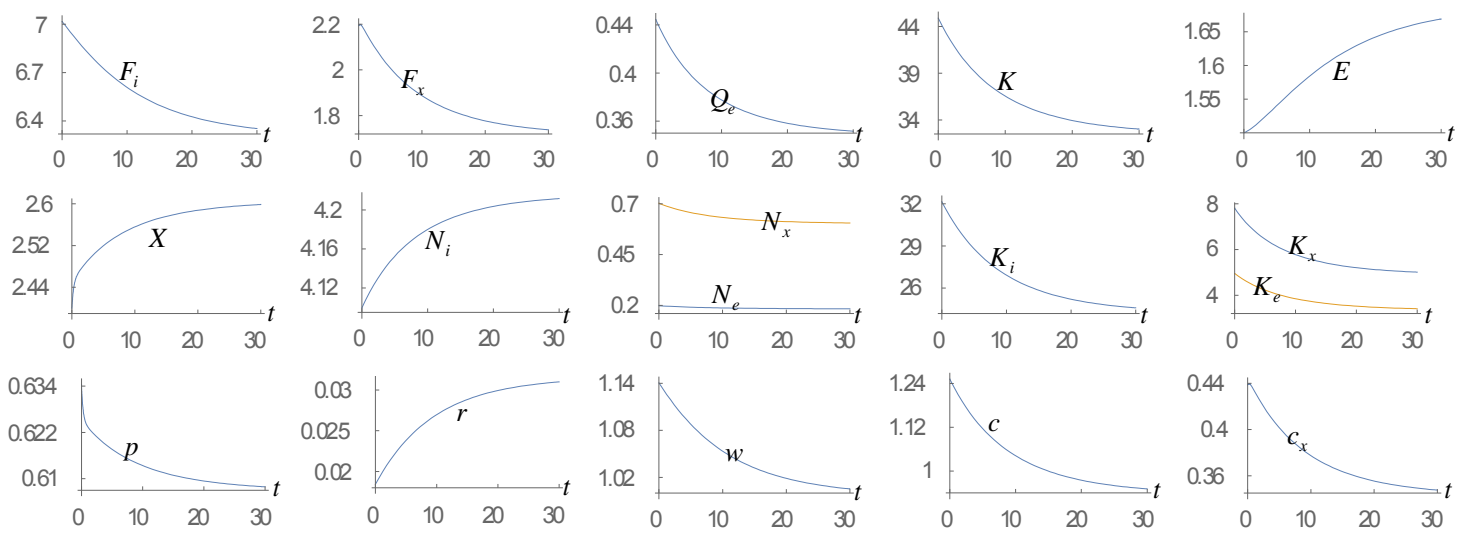

Fig. 1: Motion of the Economic System

\section{Comparative Dynamic Analysis}

We consider the parameters in (19) as the long-term average values. First, we introduce a variable $\bar{\Delta} x(t)$ to stand for the change rate of the variable $x(t)$ due to changes in the parameter value. Figure 2 shows the oscillations when the carrying capacity of the resource are specified as $\phi(t)=3+0.1 \sin (t)$.

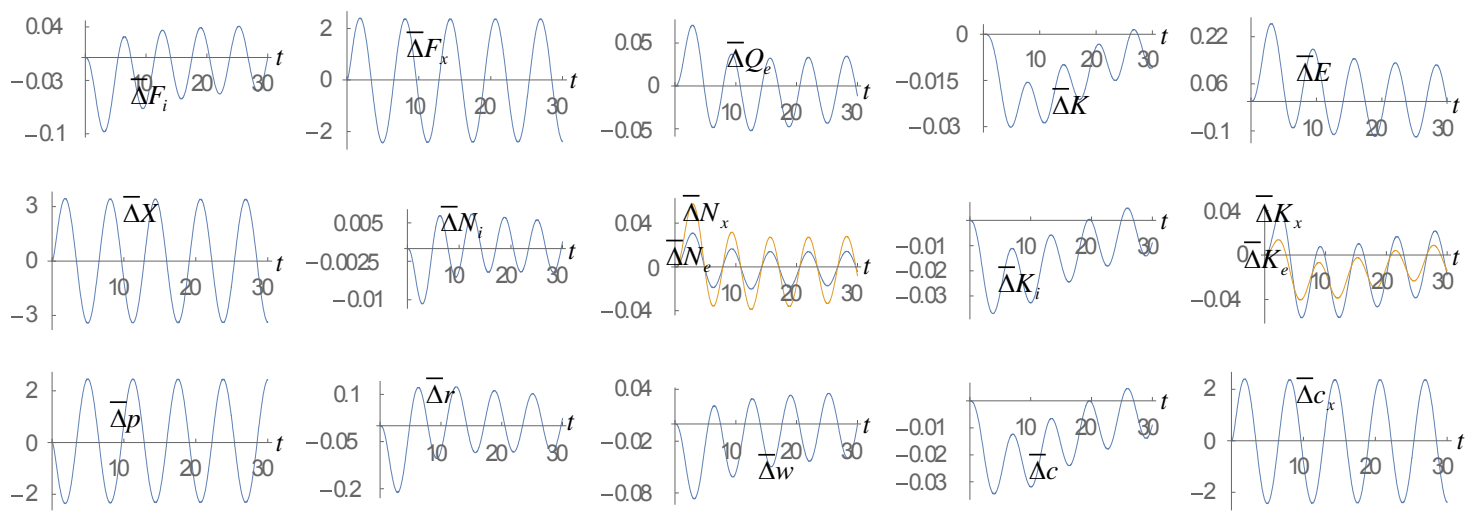

Fig. 2: Fluctuations in the carrying capacity of the resource

Figure 3 shows the oscillations when the propensity to save follows: $\lambda_{0}(t)=0.6+0.02 \sin (t)$.

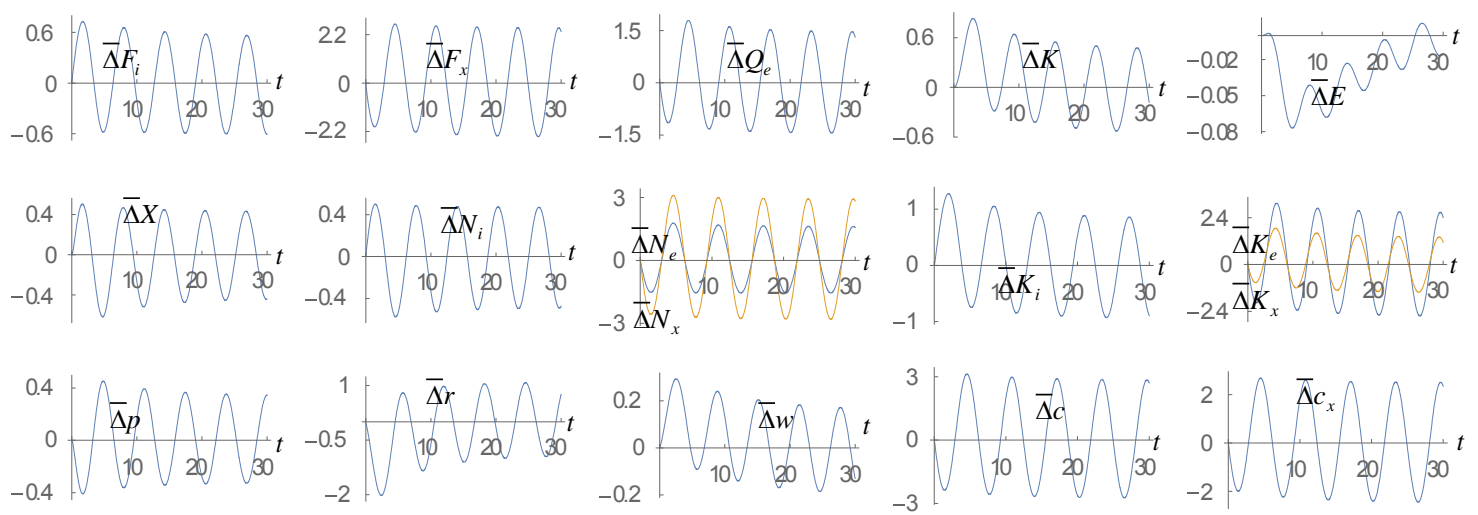

Fig. 3: Fluctuations in the propensity to save 


\section{Conclusions}

This paper identified business cycles in a dynamic economic model with endogenous physical capital, pollution, and renewable resources proposed by Zhang [1].

\section{Appendix}

The appendix shows the lemma. From (2) and (5), and (15), we obtain

$$
z \equiv \frac{r+\delta_{k}}{w}=\frac{\tilde{\alpha}_{i} N_{i}}{K_{i}}=\frac{\tilde{\alpha}_{x} N_{x}}{K_{x}}=\frac{\tilde{\alpha}_{e} N_{e}}{K_{e}}
$$

where we omit time index and $\tilde{\alpha}_{j} \equiv \alpha_{j} / \beta_{j}, j=i, x, e$. Insert (A1) in $N_{i}+N_{x}+N_{e}=N$ in (18)

Insert (A1) and (1) in (2)

$$
\frac{K_{i}}{\tilde{\alpha}_{i}}+\frac{K_{x}}{\tilde{\alpha}_{x}}+\frac{K_{e}}{\tilde{\alpha}_{e}}=\frac{N}{z} \text {. }
$$

$$
r+\delta_{k}=\frac{\alpha_{i} \bar{\tau}_{i} A_{i} \Gamma_{i} z^{\beta_{i}}}{\tilde{\alpha}_{i}^{\beta_{i}}}, w=\frac{\beta_{i} \tilde{\alpha}_{i}^{\alpha_{i}} \bar{\tau}_{i} A_{i} \Gamma_{i}}{z^{\alpha_{i}}} .
$$

From the definition of $Y_{e}$ we have

$$
Y_{e}=\tau_{i} F_{i}+\tau_{x} p_{x} F_{x}+\tau_{0} N \hat{y}+\tau_{w} N w+\tau_{k} r K
$$

where we use (11) and $\tau_{0} \equiv \tau_{c} \xi+\tau_{x} \chi$. Insert (8) in (A6)

$$
Y_{e}=\frac{\tau_{i} w N_{i}}{\beta_{i} \bar{\tau}_{i}}+\frac{\tau_{x} w N_{x}}{\beta_{x} \bar{\tau}_{x}}+\tau_{0} K+\left(\bar{\tau}_{k} \tau_{0}+\tau_{k}\right) r K+\left(\bar{\tau}_{w} \tau_{0}+\tau_{w}\right) N w,
$$

where we also use (2) and (5). From (A7), $w N_{e}=\beta_{e} Y_{e}$ and $z=\left(r+\delta_{k}\right) / w$, we have

$$
\begin{gathered}
\frac{N_{e}}{\beta_{e}}=\frac{\tau_{i} N_{i}}{\beta_{i} \bar{\tau}_{i}}+\frac{\tau_{x} N_{x}}{\beta_{x} \bar{\tau}_{x}}+\frac{\bar{\tau}_{0} K}{w}+h(z, K), \\
\bar{\tau}_{0} \equiv \tau_{0}-\left(\bar{\tau}_{k} \tau_{0}+\tau_{k}\right) \delta_{k}, h(z, K) \equiv\left(\bar{\tau}_{k} \tau_{0}+\tau_{k}\right) z K+\left(\bar{\tau}_{w} \tau_{0}+\tau_{w}\right) N .
\end{gathered}
$$

Substituting $p c_{x}=\chi \hat{y}$ in (11) into (16) yields: $\chi N \hat{y}=p F_{x}$. Insert (8) in $\chi N \hat{y}=p F_{x}$,

$$
\left(1+\bar{\tau}_{k} r\right) \chi K+\bar{\tau}_{w} w \chi N=\frac{w N_{x}}{\beta_{x} \bar{\tau}_{x}}
$$

where we also use (2) and (5). From (A9) and $z=\left(r+\delta_{k}\right) / w$, we have

$$
\frac{K}{w}+\bar{\gamma}_{k} \bar{\tau}_{k} z K+\bar{\gamma}_{k} \bar{\tau}_{w} N=\frac{\bar{\gamma}_{k} N_{x}}{\chi \beta_{x} \bar{\tau}_{x}},
$$

where $\bar{\gamma}_{k} \equiv 1 /\left(1-\bar{\tau}_{k} \delta_{k}\right)$. From (A1), (A8) and (A10), we solve

$$
\begin{gathered}
K_{e}=h_{i} K_{i}+h_{x} K_{x}+\bar{h} \\
h_{i} \equiv \frac{\tau_{i}}{\bar{\tau}_{i}} \frac{\tilde{\alpha}_{e} \beta_{e}}{\tilde{\alpha}_{i} \beta_{i}}, h_{x} \equiv\left(\tau_{x}+\frac{\bar{\tau}_{0} \bar{\gamma}_{k}}{\chi}\right) \frac{\tilde{\alpha}_{e} \beta_{e}}{\tilde{\alpha}_{x} \bar{\tau}_{x} \beta_{x}}, \bar{h}(z, K) \equiv \frac{\bar{h}_{0}}{z}+\bar{h}_{k} K, \\
\bar{h}_{0} \equiv\left(\bar{\tau}_{w} \tau_{0}+\tau_{w}-\bar{\tau}_{0} \bar{\gamma}_{k} \bar{\tau}_{w}\right) \tilde{\alpha}_{e} \beta_{e} N, \bar{h}_{k} \equiv\left(\bar{\tau}_{k} \tau_{0}-\bar{\gamma}_{k} \bar{\tau}_{0} \bar{\tau}_{k}+\tau_{k}\right) \tilde{\alpha}_{e} \beta_{e} .
\end{gathered}
$$

Substituting (A11) into (A2) and $K_{i}+K_{x}+K_{e}=K$, we get

$$
\begin{gathered}
\left(1+h_{i}\right) K_{i}+\left(1+h_{x}\right) K_{x}=K-\bar{h}, \tilde{h}_{i} K_{i}+\tilde{h}_{x} K_{x}=\tilde{h}, \\
\tilde{h}_{i} \equiv \frac{1}{\tilde{\alpha}_{i}}+\frac{h_{i}}{\tilde{\alpha}_{e}}, \tilde{h}_{x} \equiv \frac{1}{\tilde{\alpha}_{x}}+\frac{h_{x}}{\tilde{\alpha}_{e}}, \quad \tilde{h}(z, K) \equiv\left(N-\frac{\bar{h}_{0}}{\tilde{\alpha}_{e}}\right) \frac{1}{z}-\frac{\bar{h}_{k} K}{\tilde{\alpha}_{e}} .
\end{gathered}
$$


Solve (A12)

$$
\begin{gathered}
K_{i}=d_{i k} K-\frac{d_{i z}}{z}, K_{x}=d_{x k} K+\frac{d_{x z}}{z} \\
d_{i k} \equiv\left\{\tilde{h}_{x}+\frac{\left(1+h_{x}\right) \bar{h}_{k}}{\tilde{\alpha}_{e}}-\tilde{h}_{x} \bar{h}_{k}\right\} D, d_{i z} \equiv\left\{\tilde{h}_{x} \bar{h}_{0}+\left(1+h_{x}\right)\left(N-\frac{\bar{h}_{0}}{\tilde{\alpha}_{e}}\right)\right\} D \\
d_{x k} \equiv\left\{\tilde{h}_{i} \bar{h}_{k}-\frac{\left(1+h_{i}\right) \bar{h}_{k}}{\tilde{\alpha}_{e}}-\tilde{h}_{i}\right\} D, d_{x z} \equiv\left\{\left(1+h_{i}\right)\left(N-\frac{\bar{h}_{0}}{\tilde{\alpha}_{e}}\right)+\tilde{h}_{i} \bar{h}_{0}\right\} D, D \equiv \frac{1}{\left(1+h_{i}\right) \tilde{h}_{x}-\left(1+h_{x}\right) \tilde{h}_{i}} .
\end{gathered}
$$

It is straightforward to check that we proved the computational procedure in the lemma. From (2) and (5), we have $p F_{x}=\beta_{i} \bar{\tau}_{i} N_{x} F_{i} / \beta_{x} \bar{\tau}_{x} N_{i}$. Insert this equation in $\chi N \hat{y}=p F_{x}$,

$$
\left(1+\bar{\tau}_{k} r\right) K+\bar{\tau}_{w} N w=\frac{\beta_{i} \bar{\tau}_{i} N_{x}}{\beta_{x} \bar{\tau}_{x} N_{i}} \frac{F_{i}}{\chi}
$$

where we also use $y=\left(1+\bar{\tau}_{k} r\right) k+\bar{\tau}_{w} w$. From (1) and (A1), we have

$$
F_{i}=\frac{\tilde{\alpha}_{i}^{\alpha_{i}} A_{i} \Gamma_{i} N_{i}}{z^{\alpha_{i}}}
$$

Insert (A15) in (A14)

$$
\left(1+\bar{\tau}_{k} r\right) K+\bar{\tau}_{w} N w=\frac{w z K_{x}}{\tilde{\alpha}_{x} \chi \beta_{x} \bar{\tau}_{x}}
$$

where we also use (A1) and (A3). Inserting (A13) in (A16), we solve $K$ as a function of $z$ and $E$ as follows

$$
K=\Phi(z, E, t) \equiv\left(\frac{d_{x z}}{\tilde{\alpha}_{x} \chi \beta_{x} \bar{\tau}_{x}}-\bar{\tau}_{w} N\right)\left(1+\bar{\tau}_{k} r-\frac{d_{x k} w z}{\tilde{\alpha}_{x} \chi \beta_{x} \bar{\tau}_{x}}\right)^{-1} w .
$$

From (3) and (6), we have

$$
\dot{X}(t)=\bar{\Lambda}_{X}(z, X, E, t), \quad \dot{E}(t)=\bar{\Lambda}_{E}(z, X, E, t),
$$

where we do not give expressions of $\bar{\Lambda}_{X}$ and $\bar{\Lambda}_{X}$ as the expressions are tedious. From (A17) we have

$$
\dot{K}=\frac{\partial \Phi}{\partial z} \dot{z}+\frac{\partial \Phi}{\partial E} \bar{\Lambda}_{E}+\frac{\partial \Phi}{\partial t} .
$$

Multiplying the two sides of (12) with $N$ and using $s=\lambda \hat{y}$, we obtain

$$
\dot{K}=\lambda N \hat{y}(z, X, E)-\Phi(z, E)+\Phi(z, E) \frac{\dot{N}}{N} .
$$

From (A19) and (A20), we conclude the proof

$$
\dot{z}=\bar{\Lambda}_{z}(z, X, E) \equiv\left[\lambda N \hat{y}-\Phi+\Phi \frac{\dot{N}}{N}-\frac{\partial \Phi}{\partial E} \bar{\Lambda}_{E}-\frac{\partial \Phi}{\partial t}\right]\left(\frac{\partial \Phi}{\partial z}\right)^{-1}
$$

\section{Acknowledgements}

The author is grateful for the financial support from the Grants-in-Aid for Scientific Research (C), Project No. 25380246, Japan Society for the Promotion of Science.

\section{References}

[1] W.B. Zhang, "A dynamic interaction among capital, environment and renewable resources with environment and resource policies in a three-sector growth model," Malaysian Journal of Economic Studies, Vol. 49, pp. 121-139. 